\title{
JEAN DUNS SCOT SUR LA CONNAISSANCE INTUITIVE INTELLECTUELLE (COGNITIO INTUITIVA)
}

Gérard Sondag*

\section{L'aspect épistémologique et l'aspect psychologique du problème ${ }^{1}$}

Le problème de la cognitio (ou notitia) intuitiva chez Duns Scot présente deux aspects, l'un épistémologique, l'autre psychologique, qui sont différents quoique liés entre eux. Nous dirons pour commencer quelques mots du premier aspect, pour nous consacrer ensuite à l'étude du second. L'épistémologie (Duns Scot dirait 'la logique') concerne la vérité de la connaissance; la psychologie, les actes cognitifs. En faisant cette distinction, nous n'avons pas le sentiment de commettre un anachronisme car, comme avant lui Aristote, qui sépare nettement l'objet du Traité de l'âme de celui des Analytiques, Duns Scot connaît cette distinction et il en fait d'ailleurs un usage extensif, en l'introduisant jusque dans l'analyse des actes cognitifs eux-mêmes ${ }^{2}$.

Pour Aristote, au livre I des Seconds Analytiques, la connaissance scientifique, c'est-à-dire celle qui est à même de faire connaître le vrai - sachant que le vrai a son siège dans l'esprit -, doit vérifier quatre conditions, selon la description de Duns

Université Blaise Pascal/ Clermont

1 Pour les Opera Omnia, nous utilisons de préférence l'édition critique, dite édition vaticane (Rome, 1950-), notée 'Vat'.; à défaut, puisque cette édition est encore incomplète à ce jour, le reprint Olms (Hildesheim, 1969) de l'édition Wadding (Lyon, 1639), noté 'Olms', ou l'édition Vivès (Paris, 1891-95), notée 'Viv.'. Pour les Opera philosophica, nous utilisons l'édition critique, en 4 volumes, de l'Institut franciscain de l'Université Saint Bonaventure, N.Y. (1997-2004), notée 'St.-B'.

2 Voyez, par exemple, dans la question De l'image (Paris, Vrin, 1993, pp. 136-40) la distinction que fait Duns Scot entre les deux aspects des espèces intellectuelles, l'un noétique, l'autre psychologique. Voyez également le texte suivant, où le néologisme animastice qualifie l'acte de l'âme intellective en tant qu'acte psychologique.

"Et tamen substantia de se est perfectius cognoscibile, et in se et a nobis, si possemus ad illam pertingere; sed non in vita ista, ut habetur in II, 3 quaestione. Et tunc dictum Philosophi intelligitur metaphysice dictum, non animastice " (St.-B. IV, p. 119).

\begin{tabular}{|l|l|l|l|l|l|}
\hline VERITAS & Porto Alegre & v. 53 & n. 3 & jul./set. 2008 & p. 32-58
\end{tabular}


Scot dans la quatrième partie du Prologue de l'Ordinatio ${ }^{3}$. Tout d'abord, qu'elle soit une connaissance certaine, sans erreur ni doute. Deuxièmement, qu'elle ait pour objet un objet nécessaire. Troisièmement, qu'elle soit causée par une cause évidente pour l'intellect. Quatrièmement, qu'elle soit appliquée à l'objet de connaissance par syllogisme ou raisonnement syllogistique. Duns Scot reprend à son compte ces quatre conditions, mais il juge nécessaire d'aménager la caractérisation aristotélicienne de la science en la modifiant en partie. Selon lui, en effet, il faut poser qu'un objet contingent - par opposition à 'nécessaire' - peut être connu de façon certaine, sans risque d'erreur ni doute. Ainsi, l'âme se connaît elle-même, ou plutôt elle connaît ses propres actes en tant qu'elle les produit en elle-même. Pourtant, étant une créature, l'âme n'est pas nécessaire par soi mais contingente, et ses actes sont euxmêmes contingents. C'est l'influence d'Augustin qui se fait sentir ici, lequel, dans le traité De la Trinité, enseigne qu'elle "ne peut s'ignorer entièrement", c'est-à-dire méconnaître sa propre existence, "cette âme qui, lors même qu'elle se connaît ignorante d'elle-même, se connaît par le fait même"4. La deuxième condition - à savoir la nécessité de l'objet - peut être conservée s'agissant de la connaissance que Dieu a de la nature ou essence divine, laquelle est un objet par soi nécessaire, mais doit être écartée s'agissant de la connaissance que l'âme crée a d'elle-même. Enfin, puisque la connaissance que l'âme a d'elle-même, quoiqu'imparfaite, est directe ou immédiate (sachant que l'âme est toujours présente à elle-même), il suit que la quatrième condition posée par Aristote, à savoir l'inférence syllogistique ou le modus ponens, n'est pas une condition nécessaire, c'est-à-dire n'entre pas obligatoirement dans le concept d'une science parfaite, c'est-à-dire à même de faire connaître le vrai. Bien qu'à son habitude Duns Scot soit habile à montrer qu'il ne s'écarte pas d'Aristote ${ }^{5}$, il n'en demeure pas moins que le concept aristotélicien de la science (entendant par là la connaissance du vrai) se trouve modifié en profondeur. Non seulement, en effet, Duns Scot admet la possibilité d'une connaissance certaine de vérités contingentes, mais encore il pose qu'une connaissance de ce genre ne le cède en rien à celle des vérités nécessaires pour le degré de certitude ${ }^{6}$. Par 'vérité contingente', il ne faut pas entendre, bien entendu, une vérité qui peut être différente de ce qu'elle est (puisqu'une vérité différente de ce qu'elle est est une fausseté), mais une vérité qui est certaine, quoique son objet soit contingent.

3 DUNS SCOT, Prologue de l'Ordinatio, Paris, P.U.F., 1999, p. 270.

4 Tr. X, III, 5; Bibliothèque augustinienne, Desclée, 1955, t. 16, p. 131.

5 "Cependant, la connaissance des vérités contingentes est-elle ou non une science? Je réponds que si l'on prend la définition de la science donnée dans les Seconds Analytiques, au livre I, qui requiert que son objet soit nécesssaire, il ne peut exister de science de celles-ci, parce que connaître le contingent comme nécessaire, c'est ne pas le connaitre pour ce qu'il est; cependant, si l'on prend la science au sens où le Philosophe l'entend dans l'Ethique, livre VI, quand il l'oppose à l'opinion et à l'apparence, il n'est nullement impossible qu'il existe une science des vérités contingentes, puisqu'il peut exister aussi un habitus par lequel nous énonçons ce qui est vrai d'une façon déterminée" (Prologue de l'Ordinatio, p. 277).

6 Au sujet de nos actes internes, Duns Scot déclare que "beaucoup d'entre eux sont connus de façon aussi certaine que les principes premiers connus par soi", par exemple que je veille, que je pense, que j'entends, etc (Ord. I, d 3, p. 1, q 4, § 238; Vat III, p. 144). 
La première sorte ou la première espèce de connaissance est appelée par Duns Scot 'abstractive'. Ce terme ne renvoie pas à un acte d'abstraction, comparable à l'aphrairesis aristotélicienne, qui désigne un procédé intellectuel d'induction par lequel l'intellect 'abstrait', c'est-à-dire extrait une notion commune en écartant les conditions dites 'matérielles' de l'objet connu, par exemple le lieu et le moment, ou encore induit une règle générale à partir d'un ensemble plus ou moins étendu de cas particuliers. Bien que Duns Scot connaisse lui aussi ce processus d'abstraction, prise au sens d'une induction, la connaissance abstractive, telle qu'il la conçoit, se définit par le fait qu'elle fait abstraction (ou plutôt par le fait qu'elle est par nature à même de faire abstraction ${ }^{7}$ ), en les mettant entre parenthèses, de l'existence et de l'inexistence, de la présence ou de l'absence de l'objet connu. En bref, la connaissance abstractive n'est pas une connaissace 'abstraite' mais une connaissance 'indifférente' à l'égard de la présence ou de l'absence, de l'existence ou de l'inexistence de l'objet connu, en tant qu'il est connu.

L'indifférence envers la présence ou l'absence de l'objet est concédée par Aristote, puisque, comme on le sait, le Philosophe déclare que la connaissance de l'éclipse de la Lune et de ses causes est une et la même, quand une éclipse se produit et quand elle ne se produit pas. Elle ne dépend donc ni du lieu ni du moment, et reste vraie ut in pluribus. Cet exemple ou paradigme peut naturellement être généralisé. Mais Aristote aurait sans doute émis des réserves concernant l'indifférence envers l'existence ou l'inexistence de l'objet connu. Cela à juste titre, car on peut se demander quelle valeur aurait la connaissance d'un objet qui n'existe pas. Mais ce que Duns Scot a en tête est tout autre chose. Il veut dire que l'existence d'un objet n'est pas comprise dans le concept par soi de cet objet (ce qui, au demeurant, est conforme à Aristote). Et la raison en est claire. C'est que, en tant qu'il existe, un objet est constamment soumis au changement. Non seulement il se modifie sans cesse sous l'effet de l'environnement, mais encore il peut cesser d'exister absolument. Or la connaissance que nous en avons ne peut pas se modifier au gré du changement subi par l'objet, que ce changement soit accidentel ou même substantiel. De même, la signification d'un mot ne peut pas varier en fonction des modifications de l'objet signifié en tant qu'il existe in re extra, ni même selon que cet objet existe en acte ou cesse d'exister ${ }^{8}$. Car sinon, comme le dit Avicenne, il faudrait instituer à chaque fois un mot nouveau pour le signifier, ce qui est absurde, évidemment. Par conséquent, dire que la connaissance abstractive d'un objet est indifférente non seulement envers la présence ou l'absence, mais encore envers

7 Cette précision est utile, parce que les épithètes latins à suffixe en - (i)vus qualifient moins des espèces d'actes dans une typologie que des actes dont le sujet qui les accomplit est à même de recevoir l'action signifiée par le radical. Ainsi, un actus memorativus n'est pas tant un acte de mémoire qu'un acte qui fait que le sujet se souvient. La connotation performative des épithètes de ce genre est presque perdue dans les langues romanes issues du latin. Il est cependant nécessaire de s'en souvenir pour comprendre ce à quoi pense Duns Scot quand il parle d'un actus abstractivus ou d'un actus intuitivus: ce dernier n'est pas tant un acte visuel qu'un acte qui permet de voir, ou qui fait voir quelque chose.

8 Cette comparaison entre concept et mot se justifie par le fait qu'un mot est institué pour signifier un concept. 
l'existence ou l'inexistence de cet objet, ce n'est pas dire que la connaissance d'un objet inexistant est comparable à, et de même valeur que celle d'un objet existant. C'est dire que l'objet connu est le même en tant qu'il est connu ou conçu. Cette condition - 'en tant qu'il est connu ou conçu' - éclaire le propos scotiste. Car, en tant qu'il est connu, l'objet existe dans l'âme intellective, et nulle part ailleurs. C'est pourquoi, une fois acquise et devenue un habitus (un 'acquis intellectuel', selon le vocabulaire actuel de la psychologie cognitive), la connaissance d'un objet, quel qu'il soit, est identique à elle-même et demeure invariable et permanente (sous réserve des conditions physiologiques dont la mémoire dépend), que cet objet soit présent ou pas, qu'il existe ou non dans la réalité. Or cela vaut non seulement à propos des concepts simples, comme 'homme' par exemple, mais encore des concepts composés pour former des propositions nécessaires, par exemple quand on dit que 'l'homme est un être vivant doué de raison'. . Cela vaut aussi, bien évidemment, des raisonnements syllogistiques, et à plus forte raison. Car, si la vérité de la conclusion d'un syllogisme dépend de celle des prémisses, elle dépend aussi, en tant que conclusion, de la validité (Duns Scot dit 'l'évidence') de l'inférence, qui est de nature logique. Et l'on voit clairement quels dommages subirait la science si elle était assujettie à la condition de la présence et de l'existence de son objet.

A côté de cette analyse épistémologique, Duns Scot procède aussi à une caractérisation psychologique. Dans la première, il s'agit de la vérité de la connaissance; dans la seconde, de la réalité des actes cognitifs. Qu'il s'agisse bien, dans le second cas, de la réalité des actes cognitifs, on le voit à ceci que, comme il apparaîtra, Duns Scot s'efforce de démontrer l'existence d'actes intuitifs intellectuels chez l'homme pro statu isto, c'est-à-dire dans l'état présent de la nature humaine. La distinction entre les deux aspects du problème est claire également. Epistémologiquement, la connaissance intuitive se définit comme une connaissance certaine de vérités contingentes. Psychologiquement, l'acte intuitif intellectuel se caractérise par le fait qu'il atteint l'objet connu tel qu'il est en soi dans l'existence actuelle. Epistémologiquement, la connaissance abstractive se définit comme la connaissance 'indifférente' d'un objet, au sens expliqué plus haut. Psychologiquement, l'acte abstractif se caractérise plus simplement comme un acte portant sur un objet existant (existentiam determinatam), mais non pas nécessairement présent. Et, afin que la différence entre l'aspect épistémologique et l'aspect psychologique du problème ressorte plus clairement, il suffit de se rappeler que, tant pour Aristote que pour Duns Scot, les actes cognitifs sont des actes vivants ou vitaux, sachant que l'âme est vie, tandis que la connaissance n'est pas vivante ou vitale en tant que telle, mais seulement par les actes vivants qui l'accomplissent.

9 A notre avis, cela vaut aussi des propositions contingentes, telle que 'Socrate court', car cette proposition n'implique nullement l'existence de Socrate, ni le fait qu'il est actuellement en train de courir. La signification de cette expression demeure la même, que Socrate existe ou qu'il n'existe pas, qu'il courre ou qu'il soit assis. 


\section{Connaissance intuitive sensorielle et connaissance intuitive intellectuelle}

Le terme intuitivus provient du verbe latin déponent intueri, qui signifie voir, porter son regard sur ou dans quelque chose avec attention. Il semble que Duns Scot soit le premier auteur à introduire ce terme comme un terme technique dans une psychologie scientifique et expérimentale de la connaissance. "Et, pour user de termes brefs, dit-il, j'appelle 'abstractive' la première [connaissance ou intellection], - qui est connaissance de la quiddité [d'une chose], selon qu'elle fait abstraction de l'existence actuelle et de la non-existence. La seconde, c'est-à-dire celle qui est [connaissance] de la quiddité d'une chose selon son existence actuelle (ou qui est de la chose présente selon une telle existence), je l'appelle 'intellection intuitive'; non point en tant que 'intuitif' se contredistingue de 'discursif' (car, en ce sens, certaine connaissance 'abstractive' est intuitive ${ }^{10}$ ), mais en prenant 'intuitif' au sens absolu, à la façon dont on dit que nous intuitionnons une chose telle qu'elle est en soi ${ }^{11}$ ". Quelquefois, au lieu de l'expression composée cognitio intuitiva, Duns Scot emploie simplement le terme simple visio ${ }^{12}$, qui est courant chez Augustin dans un contexte comparable. A l'origine, ce terme s'applique à tout acte de la vue, mais Augustin, puis Duns Scot à la suite, le transposent du domaine des sens à celui de l'esprit. Puisque les sens perçoivent l'existence d'un objet en présence propre, l'esprit est capable, ou doit être capable (nous verrons plus bas les raisons de cette restriction, qui est plutôt une précaution de langage) de percevoir lui aussi l'existence de ce même objet.

Avant d'aller plus loin, il faut tout d'abord noter qu'au moment où Duns Scot la propose, cette notion est nouvelle dans les annales de la philosophie de l'esprit, c'est-à-dire, en termes anciens, la théorie de l'âme, et Duns Scot lui-même observe que le Philosophe n'en a rien dit. En effet, pour Aristote, la faculté qui est par nature à même de percevoir l'existence d'un objet, c'est une faculté sensorielle - par exemple la vue -, et elle seule, semble-t-il. "La notion, dit le Philosophe, a pour objet l'universel. En effet, l'être du cercle et le cercle, l'être de l'âme et l'âme sont identiques. Mais quand il s'agit d'un tout composé, par exemple ce cercle-ci ou l'un des cercles

10 Comme nous ne reviendrons pas sur ce point, qui est marginal pour le présent propos, expliquons-le aussitôt: la connaissance d'une quiddité simple, par exemple 'homme' ou 'pierre', est abstractive, parce que l'existence d'une chose n'est pas incluse dans sa quiddité. Elle peut cependant être dite 'intuitive', non pas proprement mais par opposition à 'discursive', parce qu'une quiddité est connue (du moins confusément) par un acte simple de l'intelligence, tandis qu'une proposition et, a fortiori, un syllogisme sont connus par un acte discursif. Si Duns Scot apporte cette précision, c'est afin de réserver au terme d'intuition le sens propre qu'il veut lui attribuer.

11 "Et ut brevibus utar verbis, primam voco 'abstractivam', - quae est ipsius quiditatis, secundum quod abstrahit ab actuali exsistentia et non-exsistentia. Secundam, scilicet quae est quiditatis rei secundum eius exsistentiam actualem (vel quae est rei prasentis secundum talem exsistentiam), voco 'intellectionem intuitivam'; non prout 'intuitiva' distinguitur contra discursivam (sic enim aliqua 'abstractiva' est intuitiva), sed simpliciter 'intuitivam', eo modo quo dicimur intueri rem sicut est de se" (Ord. II, d 3, p 2, q 2, § 321; Vat. VII, p. 553).

12 Par exemple, Q. in Metaph VII, q 15, § 27; St.-B. IV, p. 303: "Contra, intellectus noster habet aliquam intellectionem, quae dicitur visio, etc". 
singuliers, qu'il soit sensible ou intellectuel (j'appelle intellectuels les cercles mathématiques, et sensibles les cercles d'airain ou de bois), il n'y a pas pour eux de définition, mais ils sont connus par l'intelligence ou par le sens, c'est-à-dire quand ils sont vus en acte. Et quand ils s'écartent de l'acte [de voir], il n'est pas évident qu'ils existent ou n'existent pas, mais ils sont encore appelés [des cercles], et sont connus par une notion universelle"13. Naturellement, Duns Scot admet lui aussi que l'existence des choses sensibles est perçue par les sens, et il partage avec Aristote l'idée, clairement exposée dans le traité De l'âme, que les sens sont des facultés proprement cognitives, parce qu'ils connaissent quelque chose qui est. De là son commentaire: "Le sens a pour objet un 'tout composé', et c'est pourquoi l'acte du sens n'est pas abstractif de l'existence; l'intellection, elle, fait abstraction [de l'existence de l'objet]. Et cette condition de l'objet n'est pas seulement concomitante; c'est une condition par soi, en tant que l'objet est senti, laquelle accompagne par accident l'intellection quidditative. C'est de cette dernière qu'Aristote parle le plus souvent, car il semble qu'il n'ait rien dit de la vision intellectuelle"14. Ce texte des Questions sur la Métaphysique permet de cerner exactement la position de Duns Scot par rapport à Aristote pour ce qui est du présent propos. Le 'tout composé' signifie ici l'ensemble formé d'une nature et d'un objet singulier existant ayant cette nature. En d'autres termes, c'est un suppôt de cette nature et un suppôt existant, par exemple ce cercle-ci, présent dans la réalité (ou bien un cercle présent dans l'esprit - un cercle 'intellectuel', dit Aristote). L'existence de ce cercle-ci est perçue du sens, cela par soi et non par accident (comme si la perception de l'existence de ce cercle accompagnait seulement la perception des qualités sensibles de ce cercle). Selon Aristote, en même temps que ce cercle-ci est perçu dans son existence actuelle, une 'intellection quidditative' a lieu dans l'intellect, par laquelle la nature - non l'existence - de ce cercle-ci, singulier, est connue. Par conséquent, l'acte visuel portant sur l'objet existant en tant qu'existant, acte qui est celui du sens, d'un côté, et, de l'autre côté, l'acte de connaissance quidditative, qui est l'acte de l'intellect, sont concomitants. Mais Aristote n'attribue pas à l'intellect, par-dessus le marché, un acte 'visuel' ou 'intuitif', par lequel, en même temps que le sens le perçoit,

13 "Ratio vero <est> ipsius universalis. Circulo enim esse et circulus, et animae esse et anima idem. Simul autem <totius $>$, puta circuli huius et singularium alicuius, aut sensibilis, aut intellectualis (intellectuales autem dico, ut mathematicos, et sensibiles, ut aereos et ligneos), horum autem non est definitio, sed cum intelligentia aut sensu cognoscuntur, hoc est cum actu videntur. Abeuntes vero ex actu non palam utrum quidem sunt aut non sunt, sed semper dicuntur et cognoscuntur universalis ratione (Z, c. 10, 1036a 1-12; Aristoteles Latinus XXV, 3.2, p. 151). Remarquons qu'Aristote admet ici qu'un objet existant peut être connu comme existant de l'intelligence. Il s'agit toutefois d'un objet présent dans l'esprit ou l'imagination (ce cercle-ci mathématique auquel je pense) et non pas d'un objet présent in re extra. Reste donc que la perception d'un objet existant in re extra est dévolue uniquement au sens, selon Aristote.

14 'Sensus 'simul totius' est, et ideo actus sensus non est abstractivus ab exsistentia; intellectio autem abstrahitur. Et est ista conditio obiecti, non tantum per accidens concomitans, sed per se in quantum sensatum, per accidens autem semper concomitatur intellectionem quiditativam, de qua loquitur Aristoteles ut plurimum; quia de visione intellectuali nihil videtur locutus" (Q. in Metaph. VII, q 15, § 36; St.-B. IV, p. 307). 
l'intellect percevrait ce cercle-ci comme existant. En tous cas, dit Duns Scot, il ne semble pas que le Philosophe en ait jamais parlé. Et c'est ici précisément que se situe l'innovation scotiste, car Duns Scot va attribuer non seulement au sens mais aussi à l'intellect un acte intuitif, par lequel l'existence d'un cercle tracé sur le sol (pour reprendre l'exemple aristotélicien) est connue de l'intellect comme intellect, et non pas seulement perçue sensoriellement par le sens de la vue. Pour la suite de notre propos, deux points sont à retenir. Premièrement, Duns Scot construit la 'vision' intellectuelle à partir de la vision sensorielle. Par suite, si, d'un point de vue épistémologique, la connaissance abstractive se distingue de la connaissance intuitive comme deux façons différentes de connaître le vrai et d'égale valeur, d'un point de vue psychologique la distinction passe entre l'acte intuitif sensoriel et l'acte intuitif intellectuel. Deuxièmement, il faut retenir l'idée de la 'concomitance', car il s'avérera que l'acte intuitif et l'acte abstractif sont concomitants dans l'intellect.

Mais auparavant deux questions se posent d'elles-mêmes. Tout d'abord, pourquoi Duns Scot a-t-il éprouvé le besoin d'introduire cette innovation dans la théorie de l'âme, c'est-à-dire, en termes actuels, la philosophie de l'esprit? Ici, la réponse ne fait pas difficulté, car les motifs sont connus. En second lieu, si de tels actes intuitifs intellectuels existent, comment se fait-il que l'auteur du traité De l'âme ne se soit pas rendu compte de leur existence?

Les motifs qui conduisirent Duns Scot à poser l'existence d'une cognitio intuitiva intellectiva sont principalement d'ordre théologique et eschatologique. Raisons théologiques d'abord, parce que la connaissance que l'intellect divin a de la nature divine est nécessairement intuitive et non pas abstractive. Cela est clair, car cet objet - la nature divine - est béatifique, et béatifique infiniment. Or seul un objet existant et présent peut être béatifique, c'est-à-dire à même par nature de procurer la félicité ou le bonheur à qui le voit, par la fruition de cet objet. Cela est suffisamment clair d'après l'expérience humaine d'un objet susceptible de rendre l'homme heureux, serait-ce imparfaitement. Car c'est l'être aimé vu en présence propre qui rend heureux celui qui l'aime et non pas l'idée ou l'imagination qu'il peut en avoir (l'imagination de l'aimé(e), quand l'aimé(e) n'est pas là, est plutôt cause du désir, avec la souffrance particulière qui l'accompagne). Par conséquent, l'objet qui rend heureux infiniment est intuitionné sans fin et aimé éternellement, cela en présence propre-par nécessité. Raisons eschatologiques, ensuite. Ce qui est promis à l'homme, selon le mot de l'Apôtre ${ }^{15}$, c'est la vision de Dieu face à face (visio facialis). Sans entrer dans le détail de l'analyse (à laquelle il incombe de montrer comment la vision de l'essence divine par les Bienheureux est similaire à la vision divine de cette même essence sous certains rapports, et dissimilaire sous d'autres rapports), l'essentiel tient à ceci: cette vision, promise dans l'autre vie, est une opération de l'âme intellective, et non point une opération sensorielle - c'est évident. Mais une question se pose aussitôt: est-ce

15 Cor I, 13: Videmus nunc per speculum et in aenigmate, tunc autem facie ad faciem. Cit. in Quodl. VI, n 8; Olms XII, p. 145. 
le même intellect, tel qu'il est chez l'homme en l'état présent, qui est destiné à cette vision? Ou bien sera-ce un intellect différent, surnaturellement transformé et transfiguré? Tandis que certain docteur (Thomas d'Aquin) inclinait en faveur de la seconde réponse, pour des raisons qui lui appartiennent, Duns Scot opte résolument pour la première. Selon lui, en effet, s'il en était autrement, ce n'est pas l'homme qui sera béatifié, mais un être différent, dont nous n'avons aucune idée. Dans ce cas, à quoi bon remplir des volumes sur le sujet? La position qu'il adopte ici devait naturellement inciter Duns Scot à poser que, dès cette vie, l'intellect humain est capable d'actes intuitifs intellectuels. S'il est vrai, comme il le dit, que l'homme est capable de Dieu (capax Dei), il faut qu'en dépit des imperfections et limites qui résultent de l'état présent où est le viateur, son esprit soit ainsi fait qu'il puisse atteindre son but, si Dieu le veut. Comme les commentateurs l'ont souvent remarqué, pour Duns Scot - et, plus généralement, les penseurs franciscains -, la surnature s'édifie sur la nature sans la nier, mais en l'assumant.

En réponse à la seconde question, que Duns Scot ne s'est pas posée à lui-même, il nous semble ceci: mis à part le cas de l'objet interne, dont il est manifeste qu'il peut être intuitionné et qu'il l'est de facto, la présence en nous d'actes intuitifs intellectuels qui s'étendraient à des objets extérieurs n'est pas évidente. En tous cas, elle est moins manifeste que celle d'actes intellectuels abstractifs portant sur ces objets. En effet, par l'expérience de la réflexion, je puis être certain que je conçois le concept d'un objet. Ce concept devient alors de façon naturelle l'objet d'un acte second, différent du premier et donc, à la suite de l'actus rectus, prend place l'actus reflexivus. Les deux actes sont clairement distingués parce qu'ils sont successifs, et leurs objets différents. Cependant, quand il s'agit d'un acte intuitif - dans l'hypothèse où un tel acte existerait dans l'intellect -, la distinction entre cet acte et un acte réflexif qui porterait sur le précédent en le prenant pour objet, cette distinction est moins aisément certifiable. Et la raison en est que l'acte intuitif n'a pas de contenu quidditatif, car il se limite à saisir ou appréhender la présence de l'objet intuitionné ${ }^{16}$. Ainsi, l'objet intuitionné, en tant qu'il est intuitionné, n'est pas un quid. Considéré séparément, l'acte intuitif ne nous apprend rien sur l'objet intuitionné, sinon que cet objet est là. Un autre argument serait le suivant: tout concept métaphysique (ou de première intention) peut engendrer dans l'esprit un concept logique (ou de seconde intention). Si l'on dit que l'homme est un être vivant doué de raison (concept métaphysique, ou de première intention), il suit que l'homme est une espèce (concept logique, ou de second intention). Or, de ce qu'un objet est là, l'on ne voit pas comment il pourrait se faire qu'on en tire un concept logique, que ce soit celui de l'Individu, de l'Espèce, du Genre ou autre chose encore. L'inévidence d'actes intuitifs intellectuels s'expliquerait dans cette hypothèse par le fait qu'ici l'acte premier (ou de première intention) n'engendre pas dans l'intellect un acte second (ou de seconde

16 "Et sic exponendo, 'simul totum' apud Aristotelem non includet aliquod accidens sed tantum exsistentiam, quae non est de ratione alicuius, nec in quantum 'quid' nec in quantum 'hoc participans quid' $(0$. in Metaph. VII, q. 15, § 18; St.-B. IV, p. 300). L'existence n'est le quid de rien; un objet existant ne 'participe pas d'un quid', qui serait l'existence, puisque l'existence n'a pas de degré, n'étant pas une 'perfection'. 
intention). Ou alors, si un deuxième acte est engendré, il sera postérieur au premier mais son objet ne sera pas l'acte premier; ce sera le même objet que celui de l'acte premier. Dans l'appréhension d'un objet existant comme existant, les actes appréhensifs successifs doivent, nous semble-t-il, revenir au point de départ $d u$ premier acte, c'est-à-dire l'objet existant connu comme existant.

Quoi qu'il en soit de cette explication hypothétique, il ne fait pas de doute que Duns Scot lui-même estimait que l'existence en nous d'actes intuitifs s'étendant aux choses extérieures est moins certaine que celle d'actes abstractifs, lesquels portent sur l'objet connu en tant qu'il est connu, donc à l'intérieur de l'âme. A la question VI du Quodlibet parisien, il compare l'acte abstractif et l'acte intuitif du point de vue de la connaissance que nous en avons par l'expérience et a posteriori. Le contexte est celui de la vision béatifique, mais la distinction est générale et s'applique à la connaissance naturelle, puisque le docteur parle ici de la connaissance que nous avons des quiddités ou natures des choses sensibles: "Le premier de ces actes peut porter indifféremment sur un objet existant et non-existant, et aussi indifféremment sur un objet non réellement présent, de même que réellement présent. Nous faisons fréquemment l'expérience de cet acte en nous-mêmes, puisque nous concevons de manière équivalente les universaux, c'est-à-dire les quiddités des choses, soit que, par la nature même de la chose, ils aient une existence à l'extérieur dans quelque suppôt, soit non; et de même pour la présence et l'absence. Et cela est également prouvé a posteriori, car la science d'une conclusion ou l'intellection d'un principe restent de manière équivalente dans l'intellect, que la chose existe ou qu'elle n'existe pas, qu'elle soit présente ou absente; et l'acte par lequel la conclusion est connue et celui par lequel le principe est conçu sont possédés de manière équivalente"17. Au paragraphe suivant, il s'exprime par opposition: "Or il existe un autre acte de l'intellect, dont cependant nous ne faisons pas l'expérience en nousmêmes avec une égale certitude (non ita certitudinaliter); cependant un acte de ce genre est possible, c'est-à-dire un acte qui porte précisément sur un objet présent en tant que présent, et existant en tant qu'existant"18.

La seconde partie de ce texte a quelquefois induit certains commentateurs à croire que l'existence d'actes intuitifs intellectuels est douteuse selon Duns Scot, parce qu'il dit non ita certitudinaliter. A notre avis, elle n'est pas douteuse à ses yeux. Elle est seulement moins aisément expérimentable que l'existence en nous

17 "Unus indifferenter potest esse respectu obiecti existentis, et non existentis, et indifferenter etiam respectu obiecti, non realiter praesentis, sicut et realiter praesentis: istum actum frequenter experimur in nobis: quia universalia, sive quidditates rerum intelligimus aeque, sive habeant ex natura rei esse extra in aliquo supposito, sive non: et ita de praesentia et absentia. Et etiam hoc probatur a posteriori; quia scientia conclusionis, vel intellectus principii aeque in intellectu manet, re existente, et non existente, praesente, vel absente: et aeque potest haberi actus sciendi conclusionem, et intelligendi principium".

18 "Alius autem actus intelligendi est: quem tamen non ita certitudinaliter experimur in nobis: possibilis tamen est talis: qui, scilicet praecise sit obiecti praesentis, ut praesentis: et existentis, ut existentis" (Quodl. VI, n 8; Olms XII, p. 145). Nous rendons praecise par 'précisément', comme il est de coutume. Il faut cependant noter que ce terme ne signifie pas 'avec précision', mais plutôt 'avec distinction' ou 'séparément'. 
d'actes abstractifs, comme le montre l'expression non ita. Et voici deux arguments. Tout d'abord, nous trouvons dans les écrits de Duns Scot de nombreuses occurrences de la distinction des actes abstractifs et intuitifs, similaires à celle qui est donnée ici. Or, est-il vraisemblable que le docteur multiplie cette description s'il juge que l'un des membres de la distinction est d'existence douteuse? Et comment expliquer, dans cette hypothèse, que Duns Scot souligne fréquemment la supériorité de l'intellection intuitive sur l'abstractive ( $^{19}$ En second lieu, dans l'expression non ita certutidinaliter, l'adverbe certitudinaliter ${ }^{20}$ est associé avec experimur. Il s'agit donc d'une connaissance par expérience. Une connaissance par expérience est une connaissance par laquelle un objet - ici l'acte intuitif - est appréhendé et connu dans son existence même, et non point selon son concept. Certus ne qualifie pas l'objet, mais l'acte de celui qui fait l'expérience de l'objet. Un acte certain est tel qu'il ne donne lieu à aucun doute possible; il est indubitable ou infaillible ${ }^{21}$. Donc, ce que Duns Scot veut dire, c'est que la connaissance par expérience que nous pouvons faire en nous-mêmes de l'existence d'actes intuitifs est moins indubitable que celle que nous pouvons faire en nous-mêmes de l'existence d'actes abstractifs ${ }^{22}$. Or, si de tels actes intuitifs n'avaient pas d'existence en nous, nous ne pourrions en faire aucune expérience, ni certaine ni incertaine, ni indubitable ni non-indubitable. Car l'existence de quoi que ce soit précède toujours, par voie de nature, l'expérience que nous pouvons en faire, et de même la connaissance que nous pouvons en avoir ${ }^{23}$.

19 "- Omnis intellectio abstractiva et non-intuitiva est aliquo modo imperfecta" (Ord. I, d 2, p 2, q 1-4, § 394; Vat II, p. 352). "Nulla autem cognitio abstractiva alicuis obiecti perfectior est cognitione intuitiva" (Ord. II, d 9, q 2, § 19; Viv. XII, p. 454).

20 Cf. Niermeyer, Mediae Latinitatis Lexicon Minus, Brill, 2002, t. I, p. 226, s.u. certitudinaliter: certainement, certainly, gewiss; s. u. certitudo: certitude, certainty, Gewissheit.

21 L'on peut songer ici à la célèbre distinction cartésienne entre certitude et vérité. La connaissance de la res cogitans par elle-même, connaissance que, dans les Réponses aux objections, Descartes accepte finalement de regarder comme une 'intuition' (et non plus comme la conclusion d'un raisonnement) est certaine ou infaillible. Elle n'est pas vraie à proprement parler, puisqu'elle n'est pas susceptible d'être falsifiée, c'est-à-dire prouvée fausse. Ou bien elle a lieu, ou bien elle n'a pas lieu. Ou bien l'objet de l'intuition est saisi en totalité, ou bien il ne l'est pas du tout.

22 "Ex hoc ergo manifesto, scilicet quod intellectus potest intelligere universale, etc" (Ord. I, d 3, p. 3, q 1, §§ 348-351; Vat. III, pp. 209-211). Pour une liste des actes intellectuels dont nous faisons en nous-mêmes l'expérience, cf. Ord. IV, d 43, q 2; Olms X, pp. 24-25.

23 Concernant le terme certitudinaliter, cf. Ord. IV, d 49, q 8, n 5; Viv. XXI, p. 306: "Praeterea, si non haberemus de aliquo cognitionem intuitivam, non sciremus de actibus nostris si insunt nobis, vel non certitudinaliter (de actibus dico intrinsecis), sed hoc est falsum". Il faut reconnaitre cependant que, dans les Q. in Metaph, Duns Scot attribue la cognitio intuitiva (prima cognitio) aux sens, et la refuse à l'intellect in via: "Notandum quod in sensu est una cognitio intuitiva, primo propria; alia primo et per se propria per speciem, sed non intuitiva (...). In intellectu, notitia visionis vel intuitiva - prima cognitio - non est possibilis in via, quia nulla potentia reservans speciem vel formale principium cognoscendi in absentia obiecti potest sic cognoscere, quia illa idem habet principium re praesente et non praesente" (Q. in Metaph. II, q. 2-3, $\S \S$ 80-81; St.-B. III, pp. 224-225). Cependant, la suite (Solutio plenior quaestionis - dans l'édition critique) fait entendre un son différent: "De primo gradu, scilicet cognitionis intuitivae, an sit in intellectu in via, dubium est. Videtur tamen quod sic". La même objection est présentée à nouveau: "Sed contra: nulla potentia, retentiva speciei in absentia obiecti, cognoscit rem cognitione intuitiva, in quantum scilicet praesens est per essentiam suam; sed intellectus retinet speciem - supponatur; ergo, etc". Avec cette réponse: "Si vero teneatur quod intellectus hic [= in via] possit cognoscere intuitive, potest dici quod 
Parce que l'existence dans notre intellect d'actes intuitifs portant sur des objets extérieurs n'est pas expérimentable infailliblement, comme on vient de le voir, Duns Scot entreprend de montrer que l'existence d'actes de ce genre est 'possible'. Notez qu'ici il entend par le terme 'possible' un possible réel, et non pas un possible logique - non pas quelque chose qui peut être ou ne pas être, comme tout ce qui est simplement non-contradictoire, mais quelque chose qui est réellement possible, parce que ses causes sont réelles. Duns Scot donne une preuve qui porte sur la connaissance naturelle. Exposée sous la forme d'un syllogisme, c'est une preuve a maiori. "Preuve: toute perfection de la cognition prise absolument, qui peut appartenir à la faculté cognitive sensible peut appartenir éminemment à la faculté cognitive intellectuelle; or il est de la perfection de l'acte de connaître, en tant que cognition, d'atteindre parfaitement ce qui est connu en premier ${ }^{24}$; mais il ne l'atteint pas parfaitement quand il ne l'atteint pas en soi mais seulement dans une similitude diminuée ou dérivée de celui-ci. Or, la faculté sensitive possède cette perfection dans son acte cognitif, puisqu'elle peut atteindre l'objet en soi, en tant qu'existant et en tant que présent dans l'existence actuelle, et non pas seulement en l'atteignant de façon diminuée avec une perfection diminuée; donc cette perfection appartient à la faculté intellectuelle dans son acte cognitif; mais elle ne pourrait lui appartenir, à moins que cette faculté ne connaisse l'objet existant, et en tant qu'il est présent dans son existence propre" 25 .

Ce texte est parfaitement clair quant au raisonnement, et il suffit d'apporter en complément quelques précisions terminologiques. Tout d'abord, ce qui est appelé 'similitude diminuée ou dérivée de l'objet' est une espèce, soit sensorielle, soit intellectuelle. Les sens connaissent l'objet non seulement dans ou par une espèce sensorielle (l'image rétinienne, par exemple, species in oculo en langage scolastique),

omnem actum discretum sensus concomitatur aliquis actus intellectus circa idem obiectum; et illa intellectio est visio. Sed saltem secundum primum gradum cognitionis [i. e. gradum intuitivum], nulla substantia separata hic a nobis cognoscitur" - ce qui est évident. (Ibid. §§ 111-114; St.-B. III, pp. 231232). Des échanges dialectiques de ce genre sont fréquents dans les Q. in Metaph, où Duns Scot oppose quelquefois plusieurs solutions possibles, sans déterminer mais en indiquant quelle solution est probabilis ou probabilior, c'est-à-dire non pas 'probable' ou 'plus probable', mais 'digne d'être approuvée' ou 'plus digne d'être approuvée'. Il est manifeste qu'ici la deuxième solution est plus digne d'être approuvée, selon Duns Scot: "potest dici, etc, illa intellectio est visio". A noter pour la suite que la vision intellectuelle de l'objet existant est dite 'concomitante' avec la perception sensorielle de ce même objet.

24 Cette expression - primum cognitum - est là pour réserver le cas où l'objet est contenu éminemment dans un objet intelligible supérieur, lequel est alors ce qui est connu en premier. Mais Duns Scot déclare qu'il ne se soucie pas de cette possibilité pour le moment.

25 "Hoc probatur; quia omnis perfectio cognitionis absolute, quae potest competere potentiae cognitivae sensitivae, potest eminenter competere potentiae cognitivae intellectivae: nunc autem perfectionis est in actu cognoscendi, ut cognitio est, perfecte attingere primum cognitum: non autem perfecte attingitur, quando non in se attingitur; sed tantummodo in aliqua diminuta, vel derivata similitudine ab ipso: sensitiva autem habet hanc perfectionem in cognitione sua: quia potest obiectum attingere in se, ut existens, et ut praesens in existentia reali: et non tantum diminute attingendo ipsum in quadam perfectione diminuta: ergo ista perfectio competit intellectivae in cognoscendo: sed non posset sibi competere, nisi cognosceret exsistens, et ut in existentia propria praesens est [...] " (Quodl. VI, n 8; Olms XII, p. 145). 
dit Duns Scot, mais aussi dans son existence actuelle, ou encore en tant qu'il est présent dans l'existence actuelle. Donc, l'intellect non seulement connaît l'objet par une espèce intellectuelle, mais encore il l'atteint dans son existence actuelle, ou encore en tant que présent dans l'existence actuelle. La mineure de ce syllogisme est la règle suivante: ce qui est de la perfection d'une faculté cognitive inférieure est de la perfection d'une faculté cognitive supérieure. Cela 'éminemment', c'est-àdire a maiori. Ensuite, l'expression attingere obiectum in se est expliquée à la question XIII du même Quodlibet. C'est une relatio attingentiae alterius ut termini vel tendentiae in alterum ut terminum ${ }^{26}$ : une relation, parce que l'objet est extérieur à l'acte et à l'âme; une relation de contact, non pas réel (comme le toucher), mais intentionnel (spirituel et à distance); à l'objet comme à son terme, parce que l'objet termine l'acte. Relisons le raisonnement à partir du début: il est manifeste qu'ici Duns Scot traite de la connaissance des objets extérieurs, puisque son raisonnement prend son départ dans la connaissance sensorielle.

En second lieu, un extrait du Quodlibet XIII suffit à lever les doutes qui pourraient subsister. "Donc, dit Duns Scot, l'intellect peut exercer un acte par lequel il atteint de cette façon un objet dans son existence réelle, à tout le moins un objet qui est plus noble qu'un intellect de ce genre, ou un objet aussi noble que lui. Et si l'on admet cela pour notre intellect, à savoir qu'il peut exercer un acte cognitif de ce genre, par lequel il atteint une chose en tant qu'elle existe en soi, on peut l'admettre, pour d'égales raisons, de tout objet, quel qu'il soit, parce que notre intellect est en puissance de connaître quelque objet intelligible que ce soit"27. L'argument qui précède la conclusion introduite par ergo est le même que celui du Quodlibet V. C'est l'argument a maiori, déjà exposé, qui raisonne des sens à l'intellect. L'"objet plus noble qu'un intellect de ce genre", à savoir le nôtre, c'est manifestement l'essence divine. L' "objet qui est aussi noble que lui" ne peut être que cet intellect lui-même, puisque lui seul est aussi noble que lui-même. Le raisonnement est donc le suivant: si l'on accepte que notre intellect - ou notre âme - peut atteindre (dans l'autre vie) l'essence divine en tant qu'elle est ou existe en soi, et qu'il peut atteindre (dès cette vie) sa propre essence en tant qu'elle est ou existe en soi, il faut accepter qu'il peut atteindre (dès cette vie) quelque objet intelligible que ce soit, en tant qu'il existe en soi. Remarquons que l'inférence que fait Duns Scot n'est pas absolument claire, car l'expression pari ratione ('pour d'égales raisons'), sur laquelle repose l'inférence, peut avoir plusieurs sens. Voici comment l'on peut clarifier cette inférence: si A implique $\mathrm{B}$, la proposition équivalente à la précédente n'est pas la converse, 'non-A implique

26 Quodl. XIII, n 11; Olms XII, pp. 311-12. Duns Scot dit aussi relatio unientis formaliter in ratione medii ad terminum, ad quem unit; relationem realem et actualem ad ipsum obiectum. Nous reprendrons dans notre conclusion cette idée d'une relation d"union' réelle et actuelle de l'intellect intuitif avec son terme.

27 "Ergo intellectus potest habere actum, quo sic attingat obiectum in sua reali existentia, saltem illud obiectum quod est nobilius tali intellectu, vel aeque nobile. Et si concedatur de intellectu nostro, ipsum, scilicet posse habere talem actum cognitionis, quo attingat rem, ut existentem in se, pari ratione potest hoc concedi de quocumque obiecto: quia intellectus noster est potentialis respectu cuiuscumque intelligibilis" (Quodl. XIII, n 8 ; Olms XII, p. 309). 
non-B', mais la contrapposée, 'non-B implique non-A'. Donc, si l'on nie que notre intellect peut atteindre quelque objet intelligible que ce soit en tant qu'il existe en soi, alors l'on nie qu'il puisse atteindre un objet plus noble que lui, ou un objet aussi noble que lui, en tant que ces objets existent en soi. Mais si l'on nie que notre intellect peut atteindre quelque objet intelligible que ce soit, en tant qu'il existe en soi, il faut nier que l'intellect, en tant qu'intellect, est naturellement 'ordonné' ou 'incliné' à la connaissance de l'être tout entier ${ }^{28}$. Dans une telle éventualité, l'intellect n'a pas d'objet qui lui soit propre, distinct de celui des sens, ni par conséquent d'opération qui lui soit propre. Il n'a donc pas de raison d'exister, et l'homme n'est rien de plus qu'un animal irrationalis excellens.

En troisième lieu, Duns Scot a donné un autre argument en faveur de la cognitio intuitiva, un argument qui est de nature purement psychologique. Cet argument relève donc de la science naturelle, laquelle comprend l'âme, selon Aristote. C'est l'argument par la cognitio intuitiva imperfecta. Cet argument apparaît d'abord occasionnellement dans les Questions sur la Métaphysique sous la forme d'un contrargument dans une discussion portant sur la possibilité d'une connaissance du singulier: "Contre: notre intellect a une intellection, appelée vision, laquelle peut avoir pour objet une nature existante sans vision de la singularité, de la façon dont la vue oculaire voit un objet. Donc, notre intellect est à même de recevoir immédiatement une action de la part de la chose". Duns Scot l'admet, et ce qu'il faut retenir ici, c'est la raison pour laquelle il le fait: "Cela est admis: autrement il n'y aurait pas de mémoire dans l'intellect, en tant qu'elle porte sur quelque chose de passé. Cette mémoire, en effet, ne concerne rien d'autre, sinon quelque chose qui a été l'objet d'une vision intellectuelle". La même idée est développée au livre III de l'Ordinatio: "Ainsi également, quelque objet sensible étant présent au sens, une double connaissance peut être causée dans l'intellect sous l'effet de cet objet, l'une abstractive, par laquelle l'intellect agent abstrait l'espèce de la quiddité, en tant qu'elle est une quiddité, cela à partir de l'espèce qui est dans le fantasme [i. e. l'image mentale], laquelle représente l'objet absolument [i. e. indifféremment] et non pas en tant qu'il existe ici et à tel puis tel moment; et il peut y avoir dans l'intellect une autre connaissance, intuitive, qui coopère avec l'intellect, et celle-ci peut laisser après soi, déposée dans la mémoire intellectuelle, une connaissance intuitive habituelle, laquelle, à la différence de l'autre connaissance [i.e. abstractive] n'est pas la connaissance de la quiddité prise absolument, mais celle de l'objet qui fut connu en tant qu'existant, lorsqu'il était appréhendé dans le passé, en tant que cet objet a passé"29. Ce texte peut être lu de deux façons différentes. Soit comme une nouvelle affirmation de la distinction entre connaissance abstractive et connaissance intuitive,

28 Prologue de l'Ordinatio, op. cit., p. 123.

29 "Ita etiam praesente aliquo sensibili sensui, potest virtute illius causari in intellectu duplex cognitio, una abstractiva, qua intellectus agens abstrahit speciem quidditatis, ut quidditas est, a specie in fantasmate, quae repraesentat obiectum absolute, non ut existit hic et nunc vel tunc; et alia potest esse in intellectu cognitio intuitiva, quae cooperatur intellectui, et ab hac potest derelinqui habitualis cognitio intuitiva importata in memoria intellectiva, quae sit non quidditatis absolute, sicut fuit in alia, sed cogniti ut existens, quando in praeterito apprehendebatur, ut praeteriit. Hoc modo Christus per experientiam 
soit comme une preuve expérimentale de l'existence de cette connaissance intuitive dans l'intellect. C'est la deuxième lecture qu'il faut choisir de préférence, puisqu'une preuve vaut mieux qu'une simple affirmation. Le raisonnement se présente comme suit: d'un objet que j'ai vu, je puis me rappeler non seulement ce qu'il était, mais aussi que je l'ai vu, ou encore je puis me rappeler non seulement sa nature, mais aussi l'acte par lequel j'ai appréhendé dans le passé l'existence de cet objet quand il était présent. Comme le second souvenir est spécifiquement différent du premier, la seconde connaissance sera intuitive par opposition à la première, qui est abstractive. Intuitive, la seconde connaissance sera cependant imparfaite (puisque l'objet n'est plus là, et que seul subsiste, dans la mémoire intellectuelle, le souvenir de l'avoir vu). Or, cette connaissance intuitive imparfaite présuppose nécessairement une connaissance intuitive parfaite, quand l'objet était là, car celle-là ne peut avoir d'autre cause que celle-ci. Donc, quand l'objet est là, non seulement l'intellect forme, avec les sens, puis l'imagination, une espèce intellectuelle de la quiddité de cet objet, mais aussi, et en même temps, il reçoit un acte différent, par lequel cet objet est connu comme existant et présent. Et cet acte, c'est l'acte intuitif, qui "coopère avec l'intellect", dit Duns Scot. Qui coopère plutôt, dirons-nous plutôt, avec l'acte abstractif dans l'intellect, puisque l'un et l'autre sont des actes intellectuels. Mais peut-être objectera-t-on que la cognitio intuitiva habitualis (sive imperfecta) est déposée dans la mémoire sensorielle, et non pas dans la mémoire intellectuelle? L'on peut répondre avec Duns Scot que nous pouvons nous souvenir de l'existence d'actes internes, intellectuels ou volitifs, dont la trace est nécessairement dans la mémoire intellectuelle, et non pas sensorielle, puisque ces actes ne sont pas sensoriels ${ }^{30}$. L'on peut aussi ajouter, concernant le souvenir des objets externes, que l'imaginatio (provenant de la phantasia aristotélicienne) est une mémoire ${ }^{31}$, et non un sens, selon Aristote déjà, et qu'elle est conjuguée avec l'intellect selon Aristote encore, quoique de façon contingente, selon Duns Scot.

En conclusion de ce premier développement, il nous semble légitime d'affirmer que Duns Scot attribue à l'intellect humain, pro statu isto, une connaissance intuitive

didicisse dicitur multa, hoc est per cognitiones intuitivas, id est, illorum cognitorum quantum ad existentiam et per memorias derelictas ab eis" (Ord. III, d 14, q 3, § 7; Viv. XIV, p. 528). Bien que ce passage porte directement sur les connaissances que le Christ pouvait acquérir par expérience, les conclusions peuvent être étendues à l'homme, parce que le Christ est considéré ici dans sa nature humaine.

30 Ord. IV, d 45, q 3, n 17; Viv. XX, p. 349.

31 A cet égard, la distinction que fait Duns Scot entre cognitio abstractiva et cognitio intuitiva imperfecta sive habitualis (laquelle présuppose une cognitio intuitiva perfecta sive actualis) rappelle très nettement celle, similaire, que fait Henri Bergson entre la 'mémoire-habitude' et la 'mémoire pure', sur l'exemple fameux d'une leçon apprise par cœur. La première (souvenir de la leçon apprise) ne contient pas de référence au passé en tant que passé. La seconde fait ou peut faire revenir à l'esprit un souvenir dit 'de luxe' (celui de tel jour où, en telle circonstance, j'ai appris ma leçon). Le propos de l'un et l'autre auteur est certes différent, mais l'analogie est renforcée du fait que la'mémoire pure', involontaire, de Bergson, est plus précieuse à ses yeux que la mémoire-habitude, de même que, pour Duns Scot, la cognitio intuitiva est "plus parfaite" que la cognitio abstractiva. Cela dit, l' 'intuition' selon Bergson diffère de la 'vision' selon Duns Scot à beaucoup d'égards, bien que tous deux procèdent d'Augustin, le second explicitement, le premier implicitement. 
intellectuelle - ou visio - dont l'objet est en premier lieu les actes internes et, en second lieu, les choses extérieures. Reste maintenant à voir quels sont les rapports entre cognitio intuitiva et cognitio abstractiva dans l'acte de connaître. Notre thèse est que la cognitio intuitiva et la cognitio abstractiva sont d'espèce différente, comme Duns Scot le dit expressément. C'est pourquoi elle peuvent être séparées quand l'objet est absent. Il est donc possible d'avoir la seconde sans la première. Mais il n'est pas possible, pensons-nous, d'avoir la première sans la seconde quand l'objet est présent. Il faut donc que l'une et l'autre connaissances puissent être conjuguées ou réunies. Sur ce point, toutefois, Duns Scot s'est limité à quelques indications dispersées. Il nous incombera donc de réunir ces indications en un tout.

\section{Connaissance intuitive et connaissance abstractive (i. e. quidditative)}

Afin d'argumenter notre thèse, il faut tout d'abord revenir sur la signification exacte des termes 'intuitif' et 'abstractif', et la cerner avec plus de précision que cela était nécessaire jusqu'ici pour distinguer actes intuitifs sensoriels et actes intuitifs intellectuels. Faute, en effet, de ces nécessaires précisions, l'on risque de tomber dans l'erreur de certains interprètes, pour qui la cognitio intuitiva et la cognitio abstractiva seraient exclusives l'une de l'autre, de sorte que si l'on a l'une, l'on n'a pas l'autre, et réciproquement ${ }^{32}$.

Commençons par la première connaissance. Duns Scot n'a de cesse d'insister sur un point décisif, afin d'être bien compris. C'est que l'objet d'un acte intuitif n'est pas un objet existant en un sens non-qualifié, mais un objet existant connu ou appréhendé comme existant et présent, ou encore tel qu'il existe en soi ou dans l'existence réelle ou actuelle. Cette précision est importante, car il est possible de connaître ou de concevoir un objet existant, mais non point présent. Par exemple, moi qui suis en Auvergne, dans le centre de la France, je puis bien penser à la ville de Porto Alegre, au Brésil, dont je ne doute pas de l'existence. Mais cette pensée est toute différente de celle que je pourrais avoir de cette ville brésilienne, si j'avais la possibilité de la visiter. Car seule la seconde mérite d'être appelée une pensée intuitive. En outre, la différence est manifeste tant d'un point de vue sémantique que d'un point de vue gnoséologique. Du premier point de vue, sémantique, les expressions inquantum ou bien ut (ou bien tamquam, quoique plus rarement) s'entendent de deux façons différentes, comme on le voit sur un exemple. Si l'on dit homo inquantum homo est rationalis, l'expression inquantum homo est simplement redondante, puisque le prédicat rationalis est contenu par soi dans le sujet homo. Mais si l'on dit homo est risibilis inquantum rationalis (l'homme est capable de rire

32 Duns Scot lui-même dut rectifier (non sans mal) des interprétations erronées portant sur un cas similaire, celui de la distinction entre intellectus agens et intellectus patiens. Il n'y a pas deux intellects, explique-t-il, mais "une même entité absolue, illimitée en quelque façon", qui exerce des actes différents quoique complémentaires. Voir à ce sujet la question De l'image, op. cit., pp. 199-200. De même, en tant qu'il est une seule et même faculté, l'intellect peut exercer des actes différents, parce qu'il n'est pas limité aux actes abstractifs et à eux seuls. 
en tant qu'il possède la raison), l'expression inquantum rationalis indique la cause ou le motif pour lequel homo est risibilis, et cette indication (qui renferme en réalité un syllogisme implicite) est nécessaire, parce que risibilis n'est pas, comme la différence spécifique rationalis, une partie de l'essence de l'homme, mais seulement un accident essentiel ou accident par soi, c'est-à-dire un 'propre' selon la typologie de Porphyre. Du second point de vue, gnoséologique, l'expression inquantum, prise au second sens, indique la raison sous laquelle l'objet existant est connu, c'est-àdire en tant qu'il est à même d'exercer une action directe sur les facultés cognitives. La différence entre acte intuitif et acte abstractif ressort dès lors plus clairement: quand l'objet est présent, il est à même de 'mouvoir' les facultés cognitives, c'est-àdire à même de causer une mutation dans celles-ci. Quand il n'est pas présent, il ne peut évidemment pas mouvoir ni affecter ces facultés, et dans ce cas l'objet est connu seulement au moyen d'une espèce qui le représente (laquelle aura été acquise quand un objet de même nature a été connu en présence propre). "Ces actes cognitifs doivent donc être dits distincts, dit Duns Scot, et cela spécifiquement, à cause des raisons formelles [différentes] qui sont motrices ici et là: car dans l'acte cognitif intuitif, c'est la chose dans son existence propre qui est par soi motrice objectivement; dans l'acte abstractif, en revanche, ce qui est par soi moteur, c'est quelque chose en quoi la chose a l'être connaissable, que ce soit une cause contenant virtuellement la chose en tant que connaissable, ou que ce soit un effet, par exemple une espèce ou une similitude, qui contient de façon à le représenter ce dont elle est une similitude ${ }^{33 "}$.

Passons maitenant à l'acte abstractif. Dans son analyse psychologique, Duns Scot utilise la même expression - cognitio ou intellectio abstractiva - qu'il emploie dans l'analyse épistémologique. Mais, dans l'analyse psychologique, l'acte abstractif se réduit en réalité à un acte de connaissance quidditative, c'est-à-dire un acte par lequel est connue la nature ou quiddité d'un objet existant, mais non pas nécessairement présent. Pour désigner un tel objet, Duns Scot a une expression précise: existentiam determinatam, expression qui signifie une existence déterminée, et plus précisément un objet dans l'existence est vraie de façon déterminée. Ensuite, plusieurs textes montrent que, lorsqu'il parle de l'objet d'un acte abstractif, Duns Scot a en vue un objet existant, quoique non point nécessairement présent. "Il existe aussi, dit-il, un acte cognitif portant sur un objet, non point en tant qu'il existe en soi; mais, ou bien cet objet n'existe pas, ou bien, à tout le moins, l'acte ne porte pas sur cet objet en tant qu'il existe actuellement. Par exemple, quand on imagine une couleur, parce qu'il est possible d'imaginer une chose lorsqu'elle existe, comme

33 "Une cause contenant virtuellement, etc": c'est un habitus, ou une espèce déjà acquise; "un effet, par exemple une espèce, etc": c'est l'espèce actuellement engendrée par l'objet. "Dicentur igitur cognitiones distinctae, et hoc secundum speciem propter rationes formales motivas hinc, inde: quia cognitione intuitiva res in propria existentia est per se motiva obiective, in cognitione autem abstractiva est per se motivum aliquid, in quo res habet esse cognoscibile: sive sit causa virtualiter continens rem, ut cognoscibile, sive ut effectus, puta species, vel similitudo repraesentative continens ipsum, cuius est similitudo " (Quodl. XIII, n 10; Olms XII, p. 31. Egalement: "Actus abstractivus et intuitivus differunt specie, quia aliud et aliud est ibi movens: hic enim movet species similis rei; ibi autem movet res praesens in se" (Ord. IV, d 49, q 12, § 6; Viv. XXI, p. 442). 
lorsqu'elle n'existe pas" ${ }^{34}$. Ainsi que le montre l'exemple qu'il prend, la situation que Duns Scot a en vue est toute simple: l'on peut imaginer une couleur, alors même que cette couleur n'est pas présente à la vue. La couleur imaginée n'est donc pas une couleur qui existe ou qui n'existe pas, mais simplement une couleur existante, quoique non présente à la vue. C'est pourquoi l'imagination peut être appelée abstractive, par opposition avec le sens. Ce qui est confirmé par un autre passage du même Quodlibet XIII: "L'existence d'une chose peut également être connue par un acte cognitif abstractif. En effet, de même que je puis concevoir l'essence d'une chose, de même je puis concevoir son existence, bien qu'elle ne soit pas réellement hors de l'intellect" ${ }^{35}$. Comme le montre la fin de ce passage, Duns Scot n'a pas en tête ici le concept de l'existence en général, mais bien l'existence d'une chose déterminée. D'un point de vue épistémologique, le concept de l'existence est étranger à celui de la quiddité, cela absolument. D'un point de vue psychologique, c'est-àdire pour l'acte cognitif, il en va différemment, car l'acte abstractif peut porter sur une chose ayant une existence déterminée, même si la chose en question n'est pas présente actuellement.

Bien qu'il concerne la connaissance angélique, le troisième texte est particulièrement intéressant, parce que Duns Scot y découple et sépare l'acte intuitif d'un côté et, de l'autre côté, l'acte par lequel une 'existence déterminée' est connue: "Un Ange peut parvenir à recevoir un acte cognitif à partir des choses de trois manières [différentes] (...). Le premier acte cognitif a pour objet ce singulier-ci, en tant qu'il est 'celui-ci'. Le second acte est celui par lequel [un Ange] a une connaissance déterminée d'un existant, en tant qu'existant. Le troisième acte que les Anges acquièrent des choses est un acte cognitif intuitif. Et ce dernier n'est pas la même chose que le second, ni le premier; en effet, la cognition d'un singulier, en tant que singulier, peut être abstractive ${ }^{36}$, et de même la cognition d'un existant en tant qu'existant peut être abstractive elle aussi, car si un Ange révélait à un autre [Ange] l'existence à un moment donné d'une chose qu'il voit intuitivement, l'Ange auquel elle est révélée conçoit un existant déterminé, en tant qu'il est existant; toutefois, ce dernier n'a pas de cette chose une connaissance intuitive, car cela n'a lieu que par le fait d'un objet concourant immédiatement [à l'acte de connaître]"37.

34 "Aliqua etiam est cognitio obiecti, non ut existentis in se, sed vel obiectum non existit: vel saltem illa cognitio non est eius, ut actualiter existentis. Exemplum, ut imaginatio coloris quia contingit imaginari rem, quando non existit, sicut quando existit" (Quodl. XIII, n 8; Olms XII, p. 309).

35 "[...] Quia etiam ipsa existentia potest cognosci cognitione abstractiva. Sicut enim essentiam, sic existentiam possum intelligere, licet non sit realiter extra intellectum" (Quodl. XIII, n 10; Olms XII, p. 310).

36 "[...] Quia 'hic homo' non plus includit exsistentiam formaliter quam "homo'" (Duns Scot, Le principe d'individuation - De principio individuations, Paris, Vrin, 2005, p. 114). De même: "Non enim singulare ex se determinatur ad exsistentiam, sed abstrahit, sicut et universale" (Q. in Metaph. VII, q 15, § 18; St.-B IV, p. 300).

37 "Angelus potest proficere accipiendo cognitionem a rebus tripliciter (...) Prima cognitio est huius singularis ut hoc. Secunda cognitio est, qua habet determinatam notitiam existentiae inquantum existentia. Tertia cognitio, quam acquirunt Angeli a rebus, est cognitio intuitiva. Nec est illa eadem cum secunda, nec cum prima; cognitio enim singularis, ut singulare, potest esse abstractiva, ita etiam potest cognitio existentiae, ut existentia, quia si unus Angelus revelet alteri existentiam rei pro tali tempore, quam videt intuitive, Angelus cui revelatur cognoscit existentiam determinatam, ut existentia est; tamen non habet cognitionem intuitivam, quia hoc non est nisi per obiectum concurrens immediate" (Rep. Par. II, d 11, q 2, nn 11-12). 
Il est clair qu'ici, comme précédemment, le terme existentia ne signifie pas l'existence en général mais l'existence d'un objet déterminé. Il est manifeste également que cette description peut s'étendre de l'homme à l'ange, puisqu'il est question ici de la connaissance que les Anges "acquièrent des choses", entendant par là les choses de l'univers. L'on peut aussi penser à l'expérience humaine du témoignage. Comme le dit Duns Scot après Augustin, si je ne suis pas à même de voir par moi-même une chose ou d'assiter à un événement, je dois faire confiance à un témoin digne de foi. En pareil cas, j'apprends l'existence déterminée (c'est-à-dire déterminément vraie) de cette chose ou de cet événement, mais je n'en ai pas une connaissance intuitive. Et si l'on objecte que Duns Scot dit existentiam, et non pas existens, l'on peut répondre qu'il emploie fréquemment le premier terme pour le second, comme on le voit, entre autres choses, à sa définition de la personne (reprise littéralement de Richard de Saint-Victor: naturae intellectualis incommunicabilis exsistentia ${ }^{38}$ ). La conclusion de l'analyse est donnée par Duns Scot lui-même: quia abstractiva est aeque existentis et non existentis ${ }^{39}$. L'abstractive peut porter aussi bien sur l'objet existant que sur l'objet non-existant. Ce qui signifie, comme le montrent les textes qui précèdent, que l'abstractive a le même contenu, ou connaît la même chose, que l'objet soit là ou qu'il ne soit pas là. Par suite, la connaissance abstractive n'est pas autre chose qu'une connaissance quidditative. Cependant, même quand la chose est là, l'abstractive ne connaît pas cette chose intuitivement, car cela serait contradictoire. L'abstractive et l'intuitive diffèrent donc formellement, même quand la chose est là. Cela est nécessaire, afin que l'abstractive puisse être découplée de l'intuitive quand la chose n'est pas là. Le dernier point à examiner est donc de savoir comment l'une et l'autre peuvent être couplées et réunies quand la chose est là et connue dans son existence actuelle.

Le couplage de l'acte intuitif et de l'acte abstractif (c'est-à-dire quidditatif) quand l'objet est là peut être argumenté de trois manières au moins. Premièrement, par une analogie entre les actes des facultés cognitives inférieures (sens et imagination) d'un côté et, de l'autre côté, les actes cognitifs de la faculté supérieure (l'intellect). Deuxièmement, par la simultanéité de l'acte intuitif et de l'acte abstractif ou quidditatif dans l'intellect pris séparément et en son tout. Troisièmement, par la

38 Le terme existentia signifie l'existence, comme en français; mais, à la différence du français, il connote le fait que l'objet subsiste hors de sa cause (comme l'indique la graphie ancienne exsistentia). Cela est conforme à la conception scotiste de quelque chose d'existant, "ce qui est hors de l'âme et de sa cause". L'on voit aussi par là pourquoi, d'un objet qui existe, l'on peut dire soit existens, soit existentia. C'est que existentia n'est pas la quiddité de l'existens. De même: "Sed esse existentiae, eo modo quo distinguitur ab esse essentiae, non est ex se distinctum nec determinatum (non enim esse exsistentiae habet proprias differentias alias a differentiis esse essentiae, quia tunc oporteret ponere propriam coordinationem existentiarum aliam a coordinatione essentiarum), sed praecise determinatur ex determinatione alterius" (Ord. II, d 3, p 1, q 3, § 61; Vat VII, pp. 418-19). Sur l'interprétation philologique du verbe exsistere, voir Richard de Saint-Victor: "Quod autem dicitur exsistere, subintelligitur non solum quod habeat esse, sed etiam aliunde, hoc est ex aliquo habeat esse. Hoc enim intelligi datur in verbo composito ex adiuncta sibi praepositione. Quid est enim exsistere nisi ex aliquo sistere, hoc est substantialiter ex aliquo esse?" (La Trinité, Paris: CERF, 1959, p. 254).

39 Quodl. VI, n 8; Olms XII, p. 145. 

métaphysique universelle de non-distinction réelle d'essence et d'existence dans l'être créé ou incréé.

Commençons par l'analogie. "La seconde clarification de cette distinction se tire - par analogie - des facultés sensitives: en effet, un sens particulier connait un objet d'une manière, l'imagination d'une autre manière. En effet, un sens particulier porte sur un objet en tant qu'il est par soi et existant en soi, - l'imagination connait le même objet en tant qu'il lui est présent au moyen d'une espèce, laquelle espèce pourrait représenter l'objet même dans le cas où il ne serait pas existant et présent, en sorte que l'acte cognitif de l'imagination est abstractif par comparaison avec celui d'un sens particulier. Parce que les actes qui sont séparés dans les facultés inférieures sont unis à la fin dans les facultés supérieures, ainsi ces deux manières de sentir, qui sont séparées dans les facultés sensitives à cause de la différence des organes (puisque ce n'est pas le même organe qui est capable de recevoir comme il faut l'objet d'un sens particulier et capable de recevoir comme il faut l'objet de l'imagination), sont unies dans l'intellect, auquel - comme à une seule et même faculté - l'un et l'autre actes peuvent appartenir"40. Duns Scot raisonne ici par analogie, allant des facultés inférieures (sens et imagination) à la faculté supérieure (l'intellect). Ce texte n'est pas isolé, puisque la même analogie se rencontre ailleurs, par exemple

40 "Secunda declaratio huius distinctionis - per simile - est in potentiis sensitivis: aliter enim sensus particularis cognoscit obiectum, aliter phantasia. Sensus enim particularis, est obiecti secundum quod est per se et in se exsistens, - phantasia cognoscit idem secundum quod est praesens per speciem, quae species posset esse eius licet non esset exsistens vel praesens, ita quod cognitio phantastica est abstractiva respectu SENSUS PARTICULARIS. QUIA quae sunt dispersa in inferioribus, QUANDOQUE sunt unita in superioribus, ita isti duo modi sentiendi, qui sunt dispersi in potentiis sensitivis propter organum (quia non est idem organum, quod est bene receptivum obiecti sensus particularis et bene receptivum obiecti phantasiae), uniti sunt in intellectu, cui - ut uni potentiae - potest uterque actus competere " (Ord. II, d 3, p 2, q 2, § 323; Vat VII, p. 554). Deux remarques sur ce texte. Tout d'abord, nous modifions la ponctuation de l'édition Wadding en mettant un point entre "... sensus particularis" et "Quia ..." (mots en majuscules), faute de quoi le texte est incompréhensible, et notamment le balancement 'quia ... ita'. Deuxièmement, certain interprète conteste la validité de l'analogie faite ici par Duns Scot à cause du quandoque (mot en majuscule), probablement parce qu'il comprend cet adverbe temporel comme signifiant 'quelquefois'. En effet, si, selon cette traduction, les actes qui sont séparés dans les facultés inférieures sont 'quelquefois' réunis dans les supérieures, la conclusion aboutit seulement à un résultat contingent. De fait, lorsqu'il est pris en parallèle (quandoque ... quandoque), cet adverbe signifie 'tantôt ... tantôt'. Exemple: "Scientia autem est obiecti secundum quod abstrahit ab exsistentia actuali, alioquin scientia 'quandoque posset esse, quandoque non'" (Ord. II, d 3, p 2, q 2, § 319; Vat. VII, p. 552). Mais lorsqu'il est pris sans parallèle, il signifie 'à la fin'. Cf. Niermeyer, Lexicon Minus, op. cit., t. II, p. 1140, s. u. quandoque: enfin, finalement - at last - schliesslich. D'où notre traduction. Voyez dans le texte suivant une autre occurrence du terme quandoque, avec pour sens 'à la fin': "Quandoque autem est in actione aliqua bonitas accidentalis, et illa potest esse ab alio principio quam a quo est actio, sicut ponitur bonitas moralis in actu nostro a virtute, non a potentia tantum a quo est actio secundum substantiam; et sicut in saltando bonitas accidentalis est ab arte qua quis scit corpus apte movere, licet ipsa motio sit a virtute naturali. (Q. in Metaph IX, q 3-4, § 37; St.-B. IV, pp. 552-53). Ici, quandoque, qui est pris séparément, signifie 'à la fin': "Mais il y a à la fin dans une action une bonté accidentelle, et cette bonté accidentelle peut provenir d'un principe différent du principe de l'action. Ainsi, la bonté morale est mise dans nos actes par la vertu, et non pas seulement par la faculté d'où provient l'action selon sa substance; de même il y a dans la danse une beauté accidentelle, provenant de l'art de mouvoir le corps comme il faut, bien que le mouvement lui-même soit dû à une faculté naturelle". 
à la question XIII du Quodlibet: "Consimilis distinctio probari potest in cognitione intellectiva" ${ }^{41}$. Un sens particulier, par exemple la vue, appréhende un objet en tant qu'il est par soi ou existant en soi. L'imagination connait cet objet au moyen d'une espèce représentative, en l'occurrence une image ou phantasme. Il faut être attentif ici au fait que c'est le même objet qui est connu du sens et de l'imagination, de manière différente pour l'une et l'autre faculté, mais simultanément. En effet, pour Aristote, et pour les Scolastiques à sa suite, l'imagination n'est pas principalement la faculté de se représenter des objets en leur absence, voire d'engendrer des fictions, comme le veulent certains Modernes à la suite des Romantiques. Le rôle principal de l'imagination est de former une image en coopérant avec l'espèce sensorielle. Or elle ne peut le faire, sinon quand l'image sensorielle est présente, et donc quand l'objet senti est présent, puisque l'image sensorielle disparaît quand l'objet disparaît. En conséquence, le sens et l'imagination opèrent en même temps. Naturellement, à la différence des sens, l'imagination conserve l'espèce produite. Elle est donc à même de représenter l'objet quand celui-ci n'est plus là en le reproduisant. C'est pourquoi Duns Scot dit de cette faculté qu'elle est 'abstractive', à la différence de tous les sens particuliers. Mais il est clair que l'imagination ne pourrait représenter l'objet en son absence, ni le reproduire, si elle n'avait pas formé une espèce en même temps que le sens percevait l'existence de l'objet et les qualités sensibles de celui-ci. Nous avons donc dans cette description scotiste un exemple très clair de la façon dont une faculté intuitive (le sens) et une faculté abstractive (l'imagination) opèrent simultanément. Le fait que l'imagination est dite 'abstractive' n'empêche pas que son acte soit simultané avec celui d'une faculté intuitive, par conséquent quand l'objet est présent.

L'autre point est celui-ci: quoique simultanés, l'acte du sens et celui de l'imagination sont séparés, cela à cause de la distance des organes qui effectuent les actes en question ${ }^{42}$. Duns Scot introduit alors un argument a fortiori ou a meliori: des actes qui sont séparés dans les facultés inférieures à cause de la distance des organes sont "unis à la fin dans les facultés supérieures". Quels actes? Ce sont les actes de l'intellect, qui, par analogie, correspondent respectivement à l'acte intuitif du sens et à l'acte abstractif de l'imagination. Duns Scot parle de "deux manières de sentir". Mais cette façon de parler provient uniquement de l'analogie. En réalité puisque les actes intellectuels sont, par hypothèse, analogues aux actes du sens et de l'imagination -, les "deux manières de sentir" désignent ici les deux manières de connaître, ou les deux actes cognitifs opérés par l'intellect. Or ces actes sont unis dans l'intellect qui les opère, et non pas séparés. La raison est claire: c'est que, étant une faculté non-organique, l'intellect ne peut pas avoir un organe différent

41 Quodl. XIII, n 8; Olms XII, p. 309.

42 Quel est l'organe de l'imagination, et où se trouve-t-il? Duns Scot ne le dit pas ici. Mais il est probable qu'il suit sur ce point Aristote, lequel, au chapitre 'De la mémoire et du ressouvenir' des Parva Naturalia, pose que l'imagination est "une affection du sens commun". Bien que le sens commun n'ait pas d'organe propre et distinct des organes des sens particuliers, il existe une distance entre les organes des sens externes et le sens commun, qui est un sens interne. 
pour opérer l'un et l'autre actes différents. Mais pourquoi Duns Scot parle-t-il de "facultés supérieures"? En réalité, il n'y en a qu'une seule - c'est l'intellect ou l'âme intellective -, et si l'auteur emploie le pluriel, c'est par similitude grammaticale en raison de l'analogie. Preuve: il dit plus bas que l'un et l'autre actes peuvent appartenir à l'intellect "comme à une seule et même faculté", en laquelle ces actes sont unis parce qu'ils en proviennent comme de leur source commune. En conclusion, de même que l'acte intuitif et l'acte abstractif sont simultanés mais séparés dans les facultés inférieures, sens et imagination, de même l'acte intuitif et l'acte abstractif sont simultanés mais unis dans la faculté supérieure.

Cette doctrine paraît claire, et il nous semble que les obscurités qui l'entourent sont en réalité l'effet d'une confusion chez certains commentateurs, surtout contemporains. Certains, en effet, ont une philosophie qui veut qu'une chose ou bien est présente, ou bien représentée, et ils entendent pas là, semble-t-il, que la chose est 'rendue présente' en son absence. Or, d'une part, il est difficile de comprendre comment une chose absente peut être 'rendue présente' en son absence, sinon peutêtre de façon métaphorique. D'autre part, dans la théorie ancienne et médiévale de l'âme, non seulement il est possible, mais encore il est nécessaire qu'un objet présent soit en même temps représenté. En effet, dire qu'un objet est représentée ${ }^{43}$, c'est dire qu'il y a dans l'intellect une 'similitude' de cet objet, laquelle est engendrée aussitôt, pour peu que l'objet soit convenablement rapproché. Tout d'abord, en effet, toute substance (et toute qualité, mais nulle quantité) est une 'forme active' capable par nature d'engendrer une espèce d'elle-même dans une faculté apte à la recevoir. Ensuite, ni les sens ni l'intellect ne sont des facultés libres, car il ne leur appartient pas de recevoir ou de ne pas recevoir l'espèce engendrée. Enfin, il faut insister sur le fait que, loin de contenir une similitude de l'objet (à la façon dont un tableau contient une image, par exemple), une représentation est une similitude de l'objet. C'est là son être propre, car il existe des êtres par soi, par exemple les substances, et il existe aussi des êtres qui sont des similitudes, sensorielles ou intellectuelles. Dans ces conditions, rien ne s'oppose à ce qu'un objet présent soit représenté. Loin de là, il est nécessaire que, présent, l'objet soit représenté, c'est-à-dire engendre dans l'âme intellective cette 'similitude' de lui-même qu'est l'espèce intellectuelle. Cela est nécessaire, parce que l'objet, en tant qu'il existe in re extra, reste évidemment en dehors des sens et de l'âme. En outre, l'objet n'est pas 'rendu présent' grâce à cette similitude. Il est en réalité 'rendu sensible' s'agissant d'une espèce sensorielle,

43 Quand on dit, en français actuel, qu'un objet 'est représenté', le verbe n'a pas, grammaticalement parlant, une signification temporelle mais une signification aspectuelle, correspondant à l'accompli; en outre, 'est' n'est pas ici le verbe être, mais l'auxiliaire être (le français utilise souvent le présent passif pour exprimer l'accompli). Il en va déjà de même en latin médiéval, car obiectum repraesentatum est ne signifie pas, comme en latin classique, 'un objet a été représenté' (temps passé) mais signifie, comme en français actuel, 'un objet est représenté' (aspect accompli). Cette expression désigne un 'état' de l'objet, c'est-àdire l'état qu'il a dans l'âme (habitus en latin, hexis en grec). Enfin, pour un objet quelconque, il n'y a pas seulement deux possibilités, mais trois - être hors de l'âme, être dans l'âme, être hors de l'âme et dans l'âme. Il va de soi que seule la nature de cet objet, et non l'objet concret lui-même peut être à la fois hors de l'âme et dans l'âme, car ce n'est pas la pierre, mais la forme de la pierre qui est dans l'âme, comme l'enseigne Aristote dans le traité De l'âme. 
et 'rendu intelligible' s'agissant d'une espèce intellectuelle. C'est pourquoi, sans cette espèce interne - qu'elle soit sensorielle ou intellectuelle - l'objet, même présent, ne pourrait être ni senti ni conçu, car il est senti ou conçu par l'homme en-dedans de lui-même comme étant en-dehors de lui-même ${ }^{44}$. Toutefois, quand l'objet est présent, ce n'est pas l'espèce sensorielle qui est sentie, mais l'objet. Car je vois un objet et non pas ma vision de l'objet, parce que les sens ne peuvent réfléchir ni sur euxmêmes ni sur les espèces sensorielles. De même, quand je conçois la nature d'un objet, soit présent, soit absent, c'est cet objet que je conçois et non pas son espèce intellectuelle; cependant, comme l'intellect est une faculté réflexive, il peut se retourner sur son acte de concevoir et sur l'espèce elle-même. Ajoutons pour finir que, d'un point de vue théologique, l'intellect créé et l'intellect incréé se distinguent entre autres choses sous ce rapport, parce que ce dernier n'use pas besoin d'espèces pour concevoir les objets.

Outre l'analogie des facultés inférieures avec la faculté supérieure, Duns Scot prouve la simultanéité ou la concomitance de l'acte intuitif et de l'acte abstractif dans l'intellect pris séparément. Simultanéité, en ce sens que ces actes se produisent en même temps quand l'objet est présent. Concomitance, parce que, quoique simultanés et unis dans l'intellect, ces actes demeurent spécifiquement différents (d'où la possibilité qu'ils soient séparés, quand l'objet n'est plus présent). Au XIIIème Quodlibet, Duns Scot vient de prouver que l'acte intuitif et l'acte abstractif, autrement dit quidditatif, sont distincts dans la connaissance intellectuelle. Il rencontre alors (ou soulève lui-même) deux objections, dont la seconde dit ceci: "En outre, si deux intellections de ce genre étaient possibles pour notre intellect, alors, pour d'égales raisons, les deux pourront avoir le même objet. Je demande donc de quelle façon l'une et l'autre se distingueraient, non pas seulement par le nombre - puisque deux accidents de même espèce ne peuvent pas être présents en même temps chez le même sujet -, mais encore par l'espèce. En effet, que l'acte cognitif reçoive l'espèce soit de la faculté, soit de l'objet, alors, puisque la faculté est la même, et l'objet le même, l'on ne pourra pas poser une différence spécifique [entre les deux actes]"45. Ce que nous comprenons de la façon suivante: un acte cognitif peut être considéré comme un accident d'un sujet, en l'occurrence l'intellect ou l'âme intellective. En effet, un acte de ce genre relève de la catégorie de la qualité (plus précisément, il relève d'une sous-catégorie de la qualité, à savoir toute disposition acquise et durable). Si l'on pose deux actes cognitifs simultanés, il y aura donc deux accidents de même genre dans le même sujet, ce qui est impossible (de même qu'un homme

44 A notre avis, cette doctrine, quoique ancienne, est consonante avec les résultats expérimentaux actuels, davantage que d'autres doctrines philosophiques plus récentes, notamment la théorie phénoménologique.

45 "Praeterea. Si sint duae tales intellectiones possibiles intellectui nostro, tunc pari ratione eiusdem obiecti poterunt esse duae tales. Quaero tunc quomodo distinguerentur, non numero tantum, quia duo accidentia eiudem speciei non possunt esse in eodem subiecto simul, nec specie: quia sive actus cognitionis accipiat speciem a potentia, sive ab obiecto, cum hic sit eadem potentia, et idem obiectum, non poterit poni differentia specifica". "Ad secundum concedi potest quod sint duae cognitiones eiusdem obiecti simul, sic quod non distinguatur obiectum ab obiecto, sicut essentia ab existentia: quia licet inter ista sit aliquo distinctio obiecti, tamen non sufficiens ad propositum: quia etiam ipsa existentia potest cognosci intellectione abstractiva" (Quodl. XIII, n 9; Olms XII, p. 310). 
ne peut pas être en même temps blanc et noir). Par suite, ces deux actes ne différeront pas par le nombre, c'est-à-dire numériquement, mais seront confondus. D'autre part, ils ne peuvent pas non plus différer spécifiquement, c'est-à-dire par l'espèce. En effet, l'acte cognitif peut être causé soit par la faculté, soit par l'objet. Or, l'effet d'une cause est toujours de même espèce. Donc, que la cause soit ou bien la faculté ou bien l'objet, ni l'une ni l'autre causes ne pourront engendrer deux actes cognitifs spécifiquement différents. Par suite, quelle qu'en soit la cause, l'acte cognitif sera de même espèce ${ }^{46}$. Le docteur répond comme suit: "En réponse à la seconde objection, l'on peut admettre qu'il y ait deux cognitions d'un même objet en même temps, à condition que l'objet de l'une ne soit pas distingué de celui de l'autre comme une essence d'une existence, parce que, bien qu'il existe entre celles-ci une certaine distinction en tant qu'objets, cela n'est pas suffisant pour le présent propos, puisque l'existence elle-même peut également être conçue par intellection abstractive". Il est possible, explique Duns Scot, qu'il existe deux actes simultanés portant sur le même objet. Ces deux actes seront donc numériquement différents. Seront-ils aussi spécifiquement différents? Oui, mais non point en ce sens que l'objet du premier acte, intuitif, serait l'existence de l'objet prise séparément, et que l'objet du second, quidditatif, serait l'essence de cet objet, prise séparément de son existence. Certes, il en serait ainsi dans le cas où, dans l'objet présent, son existence et son essence seraient séparées. Dans ce cas, en effet, les deux actes seraient eux-mêmes séparés, puisque leurs objets formels respectifs seraient différents. Or, dans l'objet présent, son existence et son essence ne sont pas séparées (en langage aristotélicien, l'une et l'autre forment un simul totum). Donc, les deux actes cognitifs ne sont pas séparés, bien qu'ils soient spécifiquement différents, car le premier acte, intuitif, atteint l'objet 'en tant qu'existant', et le second connait le même objet selon sa nature ou essence. Quoique unis - et parce qu'ils portent sur un seul et même objet, et parce qu'ils proviennent d'une seule et même faculté - les deux actes sont donc spécifiquement différents ou encore formellement distincts.

Certes, concède Duns Scot pour finir, il existe une distinction entre existence et essence, du moins "en tant qu'objets". Mais cela ne veut pas dire que, dans un même objet, il existe une distinction entre son existence et son essence. Cela signifie qu'il existe une différence entre deux cas: ou bien nous avons un objet existant et présent, avec l'essence qui est la sienne (simul totum); ou bien nous avons un objet avec l'essence qui est la sienne, mais sans l'existence ou la présence actuelle. Dire que l'existence et l'essence se distinguent "en tant qu'objets", c'est dire qu'elles n'agissent pas de la même façon sur les facultés cognitives, pour la raison que l'on a vue plus haut. Cependant, conclut Duns Scot, cette distinction entre existence et essence "en tant qu'objets" n'est pas suffisante pour le présent propos, car même si une chose n'est pas présente pour moi dans son existence actuelle, je puis concevoir l'existence de cette chose en même temps que je conçois sa nature ou ce qu'elle est.

46 Cette objection ne prend pas en compte la possibilité que l'acte cognitif soit engendré à la fois par l'objet et la faculté agissent comme des causes distinctes essentiellement ordonnées, ce qui est la thèse de Duns Scot. Cf. L'image, op. cit., pp. 228-30. 
Et l'on pourrait, au demeurant, construire à partir de là un argument a maiori. S'il est effectif en effet que, d'une chose qui n'est pas présente, je puis concevoir à la fois qu'elle est ceci ou cela et qu'elle existe, a fortiori faut-il, quand cette chose est présente, que je conçoive en même temps qu'elle existe et qu'elle est ceci ou cela. Et l'on peut même ajouter cette observation psychologique bien connue, que lorsqu'une chose, surtout une chose aimée, n'est pas près de moi, la conscience que j'ai de son existence est plus vive que lorsqu'elle est là. Ainsi en va-t-il de l'homme, créature inconséquente s'il en est, qui ressent plus fortement l'existence des choses quand elles ne sont pas là, et ressent plus fortement encore le prix des choses quand elles ne sont plus. De sorte que, pour le dire avec Duns Scot, le concept abstractif de l'existence - entendant par là le concept de l'objet existant mais non présent - aurait sur l'homme un plus grand pouvoir que l'intuition actuelle de l'objet existant en tant qu'existant et présent. Cela expliquerait en outre pourquoi, comme nous l'avons suggéré plus haut, n'est pas évidente la présence en nous d'actes intuitifs intellectuels ou spirituels portant sur un objet présent en tant que présent.

Fermée cette parenthèse, il apparaît - comme le suggère l'argument qui vient d'être examiné - que la thèse gnoséologique de l'unité des actes intellectuels, intuitif et abstractif, repose en dernière instance sur une autre thèse scotiste de portée universelle, à savoir la non-distinction réelle d'essence et d'existence. Que la thèse métaphysique soit le fondement de la thèse gnoséologique, c'est clair, puisque la métaphysique, bien qu'elle soit possédée en dernier dans l'ordre de l'acquisiton de la connaissance, vient toujours en premier dans l'ordre du savoir. Entre l'existence et l'essence, l'on peut certes poser une distinction de raison, c'est-à-dire une distinction introduite par la raison, mais il est impossible d'admettre une distinction réelle, comme entre une chose et une chose (res et res, obiectum et obiectum), car deux choses réellement distinctes peuvent aller séparément: l'on aurait alors des objets existants séparés de leur nature, et des natures d'objets qui subsisteraient séparément des objets dont ces natures sont les natures ${ }^{47}$. Autant dire que nous serions alors en plein surréalisme.

\section{Ultimum dubium}

L'analyse de Duns Scot laisse subsister un dernier doute, ou plutôt une dernière interrogation, car on peut se demander si, afin de recevoir l'acte intuitif, l'intellect doit passer par l'intermédiaire des sens, de telle sorte qu'il connaîtrait l'existence d'un objet parce que les sens la connaissent d'abord, et à cette condition seulement. Ou bien faut-il penser que l'intellect est capable d'appréhender par lui-même l'objet existant, en même temps qu'il en conçoit la nature? Comme, à notre connaissance du moins, Duns Scot n'a pas envisagé cette alternative (sans doute parce qu'elle ne lui aura pas paru justifiée), il nous incombe de faire une hypothèse sur ce point (car l'interprète doit se poser quelquefois des questions que l'auteur ne se posait pas). La première solution semble pouvoir s'autoriser d'un passage du livre IV de l'Ordinatio: "Supposé en effet que l'intellect non seulement connaisse les universaux

47 “[...] Numquam esse essentiae realiter separatur ab esse existentiae "(Ord. II, d1, q 2, § 82; Vat. VII, p. 43). 
[...], mais connaisse également de façon intuitive les choses que le sens connait, parce qu'une faculté cognitive plus parfaite et supérieure dans le même [genre ${ }^{48}$ ] connaît ce qu'une faculté inférieure connaît, et aussi parce qu'elle connaît les sensations (l'un et l'autre point est prouvé par le fait que l'intellect connaît des propositions contingentes vraies, et forme à partir de celles-ci des syllogismes; or, former des propositions et faire des syllogismes est propre à l'intellect; or la vérité de ces propositions et syllogismes est relative à des objets connus intuitivement, c'est-à-dire sous la raison de l'existence, sous laquelle ces objets sont connus du sens)" ${ }^{4}$. Si, comme le dit ici Duns Scot, l'intellect "connait les sensations", l'on peut estimer qu'il connait l'existence des choses, ainsi que les vérités contingentes, par l'intermédiaire des sens, et non pas directement. Or cela reviendrait à dire, comme l'auteur semble le suggérer dans la parenthèse finale (à moins que cette parenthèse ne soit une interpolation, ce qui paraît vraisemblable ${ }^{50}$ ), que la perception de l'existence est propre au sens, et n'est donc pas propre à l'intellect, car le propre de celui-ci, c'est de former des propositions et des syllogismes. Certes, est-il dit, propositions et syllogismes peuvent porter sur des vérités contingentes, mais cellesci, ou plutôt leurs objets, seraient proprement connus du sens, et de lui seul. Mais on voit immédiatement qu'une semblable interprétation ruinerait tous les efforts de Duns Scot en faveur de la visio intellectualis et raménerait sa doctrine à une position de type aristotélicien. En réalité, l'alternative posée plus haut n'est pas fondée. Car Duns Scot dit que l'intellect "connaît de façon intuitive les choses que le sens connaît" - un point, c'est tout. Bien entendu, si l'objet n'est pas présent, il ne peut être connu intuitivement de l'intellect parce qu'il ne peut pas être connu intuitivement des sens eux-mêmes (le notion d'une cognitio intuitiva rei non-existentis est en effet une notion contradictoire $\left.{ }^{51}\right)$. Mais, quand l'objet est présent, il n'est pas connu intuitivement de l'intellect parce qu'il est connu intuitivement du sens, car cela reviendrait à faire de l'intuition sensorielle la cause de l'intuition intellectuelle. Or, il est contraire à une théorie scotiste de l'âme (et contraire déjà à la théorie aristotélicienne) de faire des actes sensoriels la cause des actes intellectuels, car alors les actes intellectuels ne

48 C'est-à-dire le genre des facultés cognitives (il y a en effet des facultés non-cognitives, par exemple la végétative ou la motrice).

49 "Supposito enim quod intellectus non tantum cognoscat universalia [...], sed etiam intuitive cognoscat illa, quae sensus cognoscit, quia perfectior et superior cognoscitiva in eodem, cognoscit illud quod inferior, et etiam quod cognoscit sensationes (et utrumque probatur per hoc quod cognoscit propositiones contingenter veras, et ex eis syllogizat; formare autem propositiones, et syllogizare proprium est intellectui; illarum autem veritas est de obiectis ut intuitive cognitis, sub ratione scilicet existentiae, sub qua cognoscuntur a sensu)" (Ord. IV, d 45, q 3, § 17; Viv. XX, p. 348 sq.).

50 Les parenthèses, ou 'postilles' que l'on trouve dans les textes scotistes sont quelquefois des notes explicatives ajoutées ultérieurement, mais conservées à bon escient par les éditeurs. Il arrive toutefois, quoique rarement, que ces ajouts gauchissent le sens du texte où ils sont inserés. Nous donnons quelques exemples de cette éventualité dans notre Introduction au Principe d'individuation, op. cit.

51 L'on peut certes concéder que, par une action sumaturelle, l'espèce d'un objet inexistant soit causée dans l'organe et la faculté visuelle - car ce n'est pas contradictoire. Mais on ne peut pas concéder que l'objet qui serait vu de cette façon serait connu par un acte intuitif - car cela est contradictoire. "Et illa cognitio [scil. intuitiva] tantum est de re praesente et sub ratione praesentis. Unde si visus posset habere speciem in absentia obiecti, numquam illa species esset principium intuitive cognoscendi" (Q. in Metaph. II, q. 2-3, § 81; St.-B. III, p. 225). 
seraient pas des actes propres de l'intellect, ayant en lui leur cause originelle - ce qui reviendrait à une conception sensualiste. Ajoutons un dernier argument de notre cru: non seulement une faculté supérieure connaît ce que connaît une faculté inférieure, mais encore elle connaît plus parfaitement ce qui est connu moins parfaitement de l'inférieure. Cela parce que dans tout ordre de perfection, la supériorité extensive (qui connaît le plus connaît le moins) implique nécessairement une supériorité intensive (le degré inférieur de connaissance est contenu éminemment dans le degré supérieur). Si donc l'intellect (qui a l'acte intuitif et l'acte quidditatif) est supérieur au sens (qui a l'acte intuitif mais non point l'acte quidditatif) d'un point de vue extensif, il suit qu'il est aussi supérieur aux sens d'un point de vue intensif, ce qui veut dire que l'intellect connait l'objet existant plus parfaitement que le sens le connait. Cette preuve a priori rejoint d'ailleurs la preuve a posteriori ou expérimentale donnée plus haut avec la cognitio intuitiva imperfecta, prouvant que l'intellect se souvient de ses actes intuitifs passés et des objets de ces actes; l'intuition parfaite et actuelle qu'il a de l'objet existant, prolongée par l'intuition imparfaite ou mémorielle qu'il a de l'objet existant quand il existait - ces deux intuitions successives dont il est capable, d'une part en tant qu'intelligence actuelle, d'autre part en tant que mémoire intellectuelle, montrent que l'intellect n'est pas limité dans ses opérations ni borné par l'instant, comme c'est le cas de l'intuition sensorielle; bien d'autres arguments pourraient être trouvés en suivant la voie de la perfection.

Pour ce qui concerne, en second lieu, l'intuition des actes internes, il est manifeste qu'ici l'acte cognitif porte directement sur l'existence de ces actes, puisque des actes de ce genre ne sont pas perçus sensoriellement, ni ne peuvent l'être. C'est d'ailleurs ce que Duns Scot lui-même déclare, en se référant à l'homme volant' d'Avicenne, ainsi qu'à Augustin: "Cela est douteux [dit-il en réponse à une objection], parce qu'assurément selon Avicenne, au livre VI des Naturalia, partie 1, et selon Augustin également, si un homme qui vivrait ne sentait jamais rien et s'il était dans l'état accompli de son âge, il saurait [cependant] qu'il vit et qu'il existe. Donc, il est certain à tout le moins que l'acte intellectuel n'est pas précédé par un acte sensoriel comme par son origine, quand bien même il pourrait prendre naissance ailleurs" 52 .

\section{Conclusions}

Simultanéité, concomitance, union - Duns Scot nous laisse sur ces mots, sans indiquer avec davantage de précision quelle sorte d'unité doit être posée entre l'acte intuitif et l'acte abstractif ou quidditatif dans l'intellect. L'on est donc en droit de faire une hypothèse. A notre avis, l'unité recherchée ne peut pas être une composition

52 "Hoc dubium est, quia forte secundum Avicennam VI Naturalium, parte 1, et secundum Augustinum, si quis viveret et nihil umquam sensisset et esset in statu perfectae aetatis, cognosceret se vivere et esse. Ergo hoc saltem certum est quod cognitionem intellectivam non praecedit sensitiva ut origo, etiam si aliunde posset oriri" (Q. in Metaph. I, q 4, § 13; St.-B. III, p. 99. Cf. Avic., De an. 1, c 1 (AviL I-III, 36-37). A noter que dans ce texte l'adverbe forte (fortè dans l'éd. Wadding) signifie 'assurément', et non pas 'peut-être'. Au sujet de ce point de sémantique du latin médiéval, voir notre article "Sur la signification du terme forte dans le latin de Jean Duns Scot", in: Duns Scot à Paris, 1302-2002, Turnhout: Brepols, 2004, pp. 151-75. 
comme celle de $a$ et de $b$, pour la raison que le premier acte et le second ne sont pas les parties d'un tout composé. L'on ne peut pas non plus regarder l'un de ces actes comme potentiel par rapport à l'autre, puisqu'ils sont tous deux actuels, du moins quand l'objet est présent et connu dans son existence propre. Or la composition des parties dans le tout et celle de l'actuel et du potentiel dans le composé sont les deux seuls types de composition connus. Peut-être peut-on suggérer alors que les deux actes sont complémentaires et qu'ils peuvent former un tout, non pas à la façon de $a$ et $b$, mais à la façon de $a$ sans $b$ et de $b$ sans $a$. Cela est possible, parce que l'acte intuitif a n'a pas, en tant que tel, de contenu quidditatif, et que l'acte abstractif $b$ n'a pas, en tant que tel, de caractère 'visuel' ou 'visionnel'. Ils ne diffèrent donc pas par l'espèce seulement, mais par le fait que l'un n'a pas ce que l'autre a, et réciproquement. Et cela suffit, semble-t-il, pour qu'ils puissent être réunis en un tout.

La seconde conclusion porte sur la 'vision', prise séparément. En élaborant, non sans mal, son concept de l'acte intuitif cognitif, Duns Scot a découvert, nous semble-t-il (et non pas inventé), quelque chose que l'esprit humain possède en lui-même, quoique le plus souvent sans le savoir. C'est que, grâce à cette vision, notre esprit est en contact avec l'ensemble des choses qui nous entourent (et même, par la mémoire intellectuelle, avec l'ensemble des choses qui nous ont entourés). Contact spirituel, et non pas sensoriel, comme on l'a vu. Contact spirituel plutôt qu'intellectuel car, bien qu'il soit le fait de l'intellect, il n'accroît pas notre connaissance des choses à la façon d'une science, et n'est donc pas cumulatif. Contact permanent - du moins dans l'état de veille -, et non pas intermittent (puisque ses causes sont elles-mêmes permanentes). Contact qui, cependant, n'est que rarement ou faiblement conscient, car les hommes perçoivent l'existence des choses environnantes pour les besoins de la vie, mais se désintéressent de l'existence des choses prises en tant qu'elles existent simplement, donc indépendamment des hommes et de leurs besoins et désirs ${ }^{53}$. Il peut se faire toutefois que l'homme prenne conscience de cette connexion qui le relie au monde environnant non seulement par la voie des sens mais aussi par l'esprit, au moyen d'un acte le plus souvent inaperçu ${ }^{54}$. Cela se produit notamment dans le phénomène de l'attention, que Malebranche a joliment nommée une "prière naturelle de l'esprit". Or l'attention est un effet de la volonté sur l'intellect, l'une et l'autre facultés étant différentes. Dans l'attention, la volonté intensifie la vision; elle ne la crée pas. La vision intellectuelle est donc déjà présente dans l'esprit, avant même la motion volontaire. C'est sur elle que repose finalement, comme sur sa possibilité a parte intellectus, l'attente des choses dernières.

53 Appliquant une distinction scotiste, l'on pourrait dire que les hommes ont pour les choses un amor commodi la plupart du temps, et un amor amicitiae plus rarement. Le premier amour, qui est plutôt un désir, est l'effet du besoin. Le second amour est la cause de la science désintéressée, de l'art, de la philosophie et de la pensée religieuse.

54 Sur la possibilité d'actes intellectuels inaperçus de l'intellect qui les opère, voir notre Duns Scot. La métaphysique de la singularité, Paris: Vrin, 2005, p. 96. 